\title{
Mrk 421 active state in 2008: the MAGIC view, simultaneous multi-wavelength observations and SSC model constrained
}

\author{
J. Aleksić1 ${ }^{1}$, E. A. Alvarez ${ }^{2}$, L. A. Antonelli ${ }^{3}$, P. Antoranz ${ }^{4}$, M. Asensio ${ }^{2}$, M. Backes $^{5}$, J. A. Barrio 2 , D. Bastieri ${ }^{6}$, \\ J. Becerra González ${ }^{7,8}$, W. Bednarek ${ }^{9}$, A. Berdyugin ${ }^{10}$, K. Berger $^{7,8}$, E. Bernardini ${ }^{11}$, A. Biland ${ }^{12}$, O. Blanch ${ }^{1}$, \\ R. K. Bock ${ }^{13}$, A. Boller ${ }^{12}$, G. Bonnoli ${ }^{3}$, D. Borla Tridon ${ }^{13}$, I. Braun ${ }^{12}$, T. Bretz ${ }^{14,26}$, A. Cañellas ${ }^{15}$, E. Carmona ${ }^{13}$, \\ A. Carosi ${ }^{3}$, P. Colin ${ }^{13}$, E. Colombo ${ }^{7}$, J. L. Contreras ${ }^{2}$, J. Cortina ${ }^{1}$, L. Cossio ${ }^{16}$, S. Covino ${ }^{3}$, F. Dazzi ${ }^{16,27}$, \\ A. De Angelis ${ }^{16}$, G. De Caneva ${ }^{11}$, E. De Cea del Pozo ${ }^{17}$, B. De Lotto ${ }^{16}$, C. Delgado Mendez ${ }^{7,28}$, A. Diago Ortega ${ }^{7,8}$, \\ M. Doert ${ }^{5}$, A. Domínguez ${ }^{18}$, D. Dominis Prester ${ }^{19}$, D. Dorner ${ }^{12}$, M. Doro ${ }^{20}$, D. Elsaesser ${ }^{14}$, D. Ferenc ${ }^{19}$, \\ M. V. Fonseca ${ }^{2}$, L. Font ${ }^{20}$, C. Fruck ${ }^{13}$, R. J. García López ${ }^{7,8}$, M. Garczarczyk ${ }^{7}$, D. Garrido ${ }^{20}$, G. Giavitto ${ }^{1}$, \\ N. Godinović ${ }^{19}$, D. Hadasch ${ }^{17}$, D. Häfner ${ }^{13}$, A. Herrero ${ }^{7,8}$, D. Hildebrand ${ }^{12}$, D. Höhne-Mönch ${ }^{14}$, J. Hose ${ }^{13}$, \\ D. Hrupec ${ }^{19}$, B. Huber ${ }^{12}$, T. Jogler ${ }^{13}$, H. Kellermann ${ }^{13}$, S. Klepser ${ }^{1}$, T. Krähenbühl ${ }^{12}$, J. Krause ${ }^{13}$, A. La Barbera ${ }^{3}$, \\ D. Lelas ${ }^{19}$, E. Leonardo ${ }^{4}$, E. Lindfors ${ }^{10}$, S. Lombardi ${ }^{6}$, A. López ${ }^{1}$, M. López ${ }^{2}$, E. Lorenz ${ }^{12,13}$, M. Makariev²1, \\ G. Maneva $^{21}$, N. Mankuzhiyil ${ }^{16}$, K. Mannheim ${ }^{14}$, L. Maraschi ${ }^{3}$, M. Mariotti ${ }^{6}$, M. Martínez $^{1}$, D. Mazin ${ }^{1,13}$, \\ M. Meucci ${ }^{4}$, J. M. Miranda ${ }^{4}$, R. Mirzoyan ${ }^{13}$, H. Miyamoto ${ }^{13}$, J. Moldón ${ }^{15}$, A. Moralejo ${ }^{1}$, P. Munar-Adrover ${ }^{15}$, \\ D. Nieto ${ }^{2}$, K. Nilsson ${ }^{10,29}$, R. Orito $^{13}$, I. Oya ${ }^{2}$, D. Paneque ${ }^{13}$, R. Paoletti ${ }^{4}$, S. Pardo ${ }^{2}$, J. M. Paredes ${ }^{15}$, S. Partini $^{4}$, \\ M. Pasanen ${ }^{10}$, F. Pauss ${ }^{12}$, M. A. Perez-Torres ${ }^{1}$, M. Persic ${ }^{16,22}$, L. Peruzzo ${ }^{6}$, M. Pilia ${ }^{23}$, J. Pochon ${ }^{7}$, F. Prada ${ }^{18}$, \\ P. G. Prada Moroni ${ }^{24}$, E. Prandini' ${ }^{6}$, I. Puljak ${ }^{19}$, I. Reichardt ${ }^{1}$, R. Reinthal ${ }^{10}$, W. Rhode ${ }^{5}$, M. Ribó ${ }^{15}$, J. Rico ${ }^{25,1}$, \\ S. Rügamer ${ }^{14}$, A. Saggion ${ }^{6}$, K. Saito ${ }^{13}$, T. Y. Saito ${ }^{13}$, M. Salvati ${ }^{3}$, K. Satalecka ${ }^{11}$, V. Scalzotto ${ }^{6}$, V. Scapin ${ }^{2}$, \\ C. Schultz ${ }^{6}$, T. Schweizer ${ }^{13}$, M. Shayduk ${ }^{13}$, S. N. Shore ${ }^{24}$, A. Sillanpää ${ }^{10}$, J. Sitarek ${ }^{9}$, D. Sobczynska ${ }^{9}$, F. Spanier ${ }^{14}$, \\ S. Spiro ${ }^{3}$, A. Stamerra ${ }^{4}$, B. Steinke ${ }^{13}$, J. Storz ${ }^{14}$, N. Strah ${ }^{5}$, T. Surić ${ }^{19}$, L. Takalo ${ }^{10}$, H. Takami ${ }^{13}$, F. Tavecchio ${ }^{3}$, \\ P. Temnikov ${ }^{21}$, T. Terzić ${ }^{19}$, D. Tescaro ${ }^{24}$, M. Teshima ${ }^{13}$, O. Tibolla ${ }^{14}$, D. F. Torres ${ }^{25,17}$, A. Treves ${ }^{23}$, M. Uellenbeck ${ }^{5}$, \\ H. Vankov ${ }^{21}$, P. Vogler ${ }^{12}$, R. M. Wagner ${ }^{13}$, Q. Weitzel ${ }^{12}$, V. Zabalza ${ }^{15}$, F. Zandanel ${ }^{18}$, and R. Zanin ${ }^{1}$
}

(Affiliations can be found after the references)

Received 8 June 2011 / Accepted 7 January 2012

\section{ABSTRACT}

Context. The blazar Markarian 421 is one of the brightest TeV gamma-ray sources of the northern sky. From December 2007 until June 2008 it was intensively observed in the very high energy (VHE, $E>100 \mathrm{GeV}$ ) band by the single-dish Major Atmospheric Gamma-ray Imaging Cherenkov telescope (MAGIC-I).

Aims. We aimed to measure the physical parameters of the emitting region of the blazar jet during active states.

Methods. We performed a dense monitoring of the source in VHE with MAGIC-I, and also collected complementary data in soft X-rays and optical-UV bands; then, we modeled the spectral energy distributions (SED) derived from simultaneous multi-wavelength data within the synchrotron self-Compton (SSC) framework.

Results. The source showed intense and prolonged $\gamma$-ray activity during the whole period, with integral fluxes $(E>200 \mathrm{GeV})$ seldom below the level of the Crab Nebula, and up to 3.6 times this value. Eight datasets of simultaneous optical-UV (KVA, Swift/UVOT), soft X-ray (Swift/XRT) and MAGIC-I VHE data were obtained during different outburst phases. The data constrain the physical parameters of the jet, once the spectral energy distributions obtained are interpreted within the framework of a single-zone SSC leptonic model.

Conclusions. The main outcome of the study is that within the homogeneous model high Doppler factors $(40 \leq \delta \leq 80)$ are needed to reproduce the observed SED; but this model cannot explain the observed short time-scale variability, while it can be argued that inhomogeneous models could allow for less extreme Doppler factors, more intense magnetic fields and shorter electron cooling times compatible with hour or sub-hour scale variability.

Key words. radiation mechanisms: non-thermal - BL Lacertae objects: individual: Mrk 421

\section{Introduction}

Blazars, a common term used for flat-spectrum radio quasars (FSRQ) and BL Lacertae objects, constitute the subclass of active galactic nuclei (AGN) that is most commonly detected in the very high energy (VHE, $E>100 \mathrm{GeV}$ ) $\gamma$-ray band. In these sources the dominant radiation component originates in a relativistic jet pointed nearly toward the observer. The double-peaked spectral energy distribution (SED) of blazars is attributed to a population of relativistic electrons spiraling in the magnetic field of the jet. The low-energy peak is commonly thought to be caused by synchrotron emission, because of its spectrum and polarization. The second, high energy peak is attributed to inverse Compton scattering of low-energy photons in leptonic acceleration models (Maraschi et al. 1992; Dermer \& Schlickeiser 1993; Bloom \& Marscher 1996). 
Alternative models invoking a relevant contribution from accelerated hadrons can also sufficiently describe the observed SEDs and light curves (Mannheim 1993; Mücke et al. 2003; but see Sikora et al. 2009 on FSRQs).

Blazars are highly variable in all wavebands and the relation between variability in different bands is a key element in distinguishing between different models. For instance, homogeneous leptonic models predict correlated variability between, e.g., $\mathrm{X}$ rays and $\gamma$ rays, which is already observed in high-frequency peaked BL Lacs (HBL; see e.g. Fossati et al. 2008 on Mrk 421 itself). On the other hand, phenomena such as the "orphan" flare from 1ES1959+650 reported in Krawczynski et al. (2004) or the ultrafast ( hundreds of seconds) events occasionally observed in some sources (e.g. Aharonian et al. 2007; Albert et al. 2007b) are harder to explain whithin this frame.

Among blazars, HBLs are the most often observed subsample in the VHE domain, because the high-energy bump peaks at $\mathrm{GeV}-\mathrm{TeV}$ energies, while the low-energy peak is located at UV to X-rays energies (Padovani 2007). This makes HBLs, such as Mrk 421, ideal targets for sensitive, low-energy threshold imaging air Cherenkov telescopes (IACT) such as MAGIC-I, in combination with soft X-ray telescopes, which observe the synchrotron bump instead; this combination of instruments samples the source SED and unravel the regions of the two peaks, the most valuable tracers of the source state.

Mrk 421 is one of the closest $(z=0.031$, de Vaucouleurs et al. 1991) and brightest extragalactic TeV sources; therefore it was the first to be detected (Punch et al. 1992) and remains one of the best studied. The VHE integral flux can vary from a few tenths to a few Crab Units (e.g., see Donnarumma et al. 2009; Hsu et al. 2009; or Pichel 2009), on time scales as short as 15 min (Gaidos et al. 1996). The $v F(v)$ distribution of the emitted photons follows the standard "double-bumped" shape, but varies significantly from low-activity states to the most intense flares, on time scales that in X-rays can be of few hours (Ushio et al. 2009). The low-energy bump peaks in the $0.1-10 \mathrm{keV}$ range (see e.g. Fossati et al. 2008), as is usual for HBLs; the maximum of the high-energy bump is usually found below $100 \mathrm{GeV}$, but can also move around according to the state of the source, usually following a "harder-when-brighter" behavior (Krennrich et al. 2002; Fossati et al. 2008; Acciari et al. 2011; Albert et al. 2007a) analogous to that traced by the X-ray emission (e.g. Brinkmann et al. 2005; Tramacere et al. 2007, 2009).

This peculiar SED shape favors multi-wavelength (MWL) studies that exploit the MAGIC-I sensitivity and low-energy threshold in VHE, and soft X-ray telescopes. MAGIC-I can detect Mrk 421 at the $5 \sigma$ level with exposures as short as a few minutes, depending on the source brightness. In the soft X-ray domain the All-Sky Monitor (ASM) onboard the Rossi X-ray Timing Explorer (RXTE) can continuously provide a daily averaged flux, while the X-Ray Telescope (XRT) onboard the Swift satellite can observe the source with far better precision and energy resolution in $\sim 1 \mathrm{ks}$ targeted exposures. From the observation of the source spectrum in both X-ray and VHE a unique set of physical parameters that describe the source can be derived within a single-zone synchrotron self-Compton (SSC) model, following Tavecchio et al. (1998). An SSC modeling of the SED of Mrk 421 has been already performed in the past (see e.g. Bednarek \& Protheroe 1997; Tavecchio et al. 1998; Maraschi et al. 1999; or the more recent Fossati et al. 2008).

Lately automated $\chi^{2}$ minimization procedures (Finke et al. 2008; Mankuzhiyil et al. 2011) have been applied.

The main limitations to previous works came from the use of the former IACTs such as Whipple (e.g. Fossati et al. 2008) or HEGRA (e.g. Takahashi et al. 2000), which were characterized by a higher energy threshold and worse sensitivity at VHE. This in turn led to poor sampling of the IC peak region, basically limited to the less informative, steeply decaying hard energy tail of the bump; moreover, integration over different nights of observation was commonly needed to obtain a significant VHE spectrum, thus yaveraging out the SED evolution, such as in Maraschi et al. (1992). In 2008 Mrk 421 went through a long and intense outburst phase, characterized by VHE fluxes quite constantly above the Crab level and superimposed shorter and brighter flares; a remarkably dense follow-up of this evolution was possible in optical, X-rays and VHE with MAGIC-I. The main outcome of this campaign is that eight tightly contemporary observations of Mrk 421 in optical, X-rays and VHE $\gamma$-rays of active states could be achieved. This allowed the reconstruction and modeling of the optical-UV/X-ray/TeV MWL SED of Mrk 421 on short time scales $(\sim 1 \mathrm{~h})$, the main improvement in this work with respect to the past literature on the subject. A similar approach was followed, for the same Mrk 421, by the VERITAS Collaboration (Acciari et al. 2011).

The paper is structured as follows: in Sect. 2 we report on the observations and data analysis; in Sect. 3 we report the VHE light curve and spectra, and complementary results in $\mathrm{X}$ rays and optical-UV band; in Sect. 4 we build the SED of Mrk 421 in the eight states for which a set of simultaneous MWL data was available, which we model in the framework of a standard one-zone SSC model; finally we discuss the results in Sect. 5.

\section{Observations and data analysis}

The timespan of the MAGIC VHE observations of Mrk 421 reported here began in December 2007 and ended in June 2008. Contemporary data from other instruments are considered for the MWL analysis, namely soft X-ray data from RXTE/ASM, and Swift/XRT, optical-UV data from Swift/UVOT and optical $R$-band data from the Tuorla Observatory. A summary description of the instruments, the datasets and the analysis follows.

\subsection{MAGIC-I VHE observations}

MAGIC $^{1}$-I (formerly MAGIC) is an IACT located on the western Canarian island of La Palma, at the Observatory of Roque de Los Muchachos (28.75 $\mathrm{N}, 17.89^{\circ} \mathrm{W}, 2225 \mathrm{~m}$ a.s.1.). With its tessellated parabolic mirror $(D=17 \mathrm{~m}, f / D=1)$, it has been the largest single-dish IACT in operation from late 2004 until the advent in 2009 of MAGIC-II, a twin (but substantially improved in many respects) telescope; since then the two telescopes are operated as a stereo IACT system (MAGIC Stereo). Its $234 \mathrm{~m}^{2}$ surface allowed for the lowest energy threshold among IACT systems at that time: the trigger threshold of the telescope at the epoch of this campaign reached as low as $60 \mathrm{GeV}$ for observations close to the zenith in optimal conditions. A detailed description of the telescope and data analysis can be found in dedicated papers (e.g. Baixeras et al. 2004; Cortina et al. 2005; Albert et al. 2008a). All MAGIC-I observations considered for the present study were performed following a major hardware upgrade (Goebel et al. 2008) that was completed in February 2007, which enhanced the time sampling capability of the data acquisition (DAQ) from $300 \mathrm{MHz}$ to $2 \mathrm{GHz}$. This allowed a better rejection of the night sky background (NSB)

\footnotetext{
${ }^{1}$ Major Atmospheric Gamma-ray Imaging Telescope.
} 
and introducing new refined analysis techniques (Tescaro et al. 2008) based on the time properties of Cherenkov signals, which lowered the integral sensitivity for point sources down to $1.6 \%$ of the Crab Nebula flux (for a $5 \sigma$ significant detection in $50 \mathrm{~h}$, above $280 \mathrm{GeV}$; Aliu et al. 2009).

During the observation period considered here, MAGIC observed Mrk 421 for a total of 81 nights, with exposure times ranging from $\sim 20$ to $\sim 240 \mathrm{~min}$. This comprised both short untriggered observations, aimed to an unbiased sampling of the source state (studied in detail in Wagner et al. 2011), and deeper extended observations of peculiar states, triggered either by the former or by external alerts from other bands. All observations were performed in the false-source tracking ("wobble", Fomin et al. 1994) mode. The method consists of alternatively tracking two positions in the sky that are symmetrical with respect to the source nominal position and $0.4^{\circ}$ away from it.

The MAGIC-I data were analyzed using the standard analysis chain described in Albert et al. (2008a,c) and Aliu et al. (2009). Preliminary quality checks were performed to exclude poor quality data, such as those owing to bad weather or occasional technical problems. Furthermore, the dataset was restricted to observations performed under dark conditions, and in the range of zenith angle ranging from $\sim 5^{\circ}$ at culmination to $46^{\circ}$. A cleaning algorithm involving the time structure of the shower images was then applied, which further selected the events and removed the NSB contribution to the images. Surviving images were parametrized in terms of the extended set of Hillas parameters (Hillas 1985) described in the mentioned literature. To suppress the unwanted background showers produced by charged cosmic rays, a multivariate classification method known as random forest (RF, Breiman 2001) was implemented and applied (Albert et al. 2008b). An analogous procedure allowed the estimation of the energy of the primary $\gamma$-rays. The signal extraction was performed by applying cuts in the Size, HadronNess and Alpha parameters described in the aforementioned literature. In particular the SizE cut, which we set to reject events with less than 150 photoelectrons of total charge, implied an energy threshold $\sim 140 \mathrm{GeV}$ in the present analysis. A total excess of $\sim 48 \times 10^{3}$ events from the selected $\sim 60 \mathrm{~h}$ of observation was detected. The whole analysis procedure was validated step by step on compatible datasets from observations of the Crab Nebula.

\subsection{Optical, UV and X-ray observations}

The Swift satellite (Gehrels et al. 2004) is a NASA mission, launched in 2003, devoted to observations of fast transients, namely prompt and afterglow emission of gamma-ray bursts. These are detected with the monitoring coded mask Burst Alert Telescope (BAT, Barthelmy et al. 2005) which is sensitive to 15-150 keV X-rays and covers a wide field of view (FoV) with a resolution of few arcminutes, and then rapidly targeted with the two co-aligned pointing instruments, XRT (Burrows et al. 2005) and Ultra-Violet Optical Telescope (UVOT, Roming et al. 2005).

The fast repositioning capability of the spacecraft allows snapshots of variable sources with little overheads. For Mrk 421, observations lasting $\sim 1 \mathrm{ks}$ allow the derivation of a detailed $\mathrm{X}$-ray spectrum and multi-filter optical-UV photometry because of the sensitivity of the targeted instruments and the brightness of the source.

Swift/XRT is a Wolter type-I grazing incidence telescope, with $110 \mathrm{~cm}^{2}$ effective area, 23.6' FoV and 15" angular resolution, sensitive in the $0.2-10 \mathrm{keV}$ energy band. During the MAGIC campaign the instrument performed 43 targeted X-ray observations of Mrk 421 of typical exposure times
1-2 ks. Swift-XRT data were reduced using the software distributed with the heasoft 6.3.2 package by the NASA High Energy Astrophysics Archive Research Center (HEASARC). The xrtpipeline was set for the photon counting or window timing modes and single pixel events (grade 0) were selected.

UVOT is a $30 \mathrm{~cm}$ diffraction-limited optical-UV telescope, equipped with six different filters, sensitive in the 1700-6500 wavelength range, in a $17^{\prime} \times 17^{\prime}$ FoV. Unfortunately, during the January 2008 campaign UVOT did not observe the source, so that the UVOT datasets were fewer than the XRT pointings and therefire five out of the eight datasets studied in Sect. 4) have no contemporary UVOT observations. Therefore we restricted the analysis of UVOT data to the three observations simultaneous with MAGIC-I performed on February 11 and April 2 and 3 with the UV filters alone. The analysis was performed with the uvotimsum and uvotsource tasks with a source region of $5^{\prime \prime}$, while the background was extracted from a source-free circular region with radius equal to $50^{\prime \prime}$ (it was not possible to use an annular region because of a nearby source). The extracted magnitudes were corrected for Galactic extinction using the values of Schlegel et al. (1998) and applying the formulae by Pei (1992) for the UV filters, and eventually were converted into fluxes following Poole et al. (2008).

The ASM onboard RXTE (Bradt et al. 1993) is sensitive enough to set one point per day from Mrk 421, which means a poorer precision, but denser coverage than Swift/XRT.

The publicly available ASM data products were taken from the results provided by the ASM/RXTE teams at MIT and at the RXTE SOF and GOF at NASA's GSFC.

The Tuorla Observatory constantly monitors the MAGIC VHE (known or potential) target sources, with the $35 \mathrm{~cm}$ remotely operated Kungliga Vetenskaplika Academy (KVA) optical telescope that is also located at Roque de los Muchachos and with a $103 \mathrm{~cm}$ telescope located at Tuorla, Finland. During the period of the MAGIC-I observations, 117 photometric measurements of Mrk 421 were obtained in the Johnson $R$-band. The optical data were reduced by the Tuorla Observatory as described in Nilsson et al. (2007). The light contribution from the host galaxy and nearby companion galaxy $\left(F_{\mathrm{h}+\mathrm{cg}}=8.07 \pm 0.47 \mathrm{mJy}\right)$ was subtracted from the measured fluxes.

\section{Results}

\subsection{MAGIC-I VHE light curves}

The night-averaged integral flux above a conservative threshold of $200 \mathrm{GeV}$ was calculated for each of the 66 nights with datasets that survived the quality cuts. The VHE light curve of Mrk 421 along the campaign is plotted in the top panel of Fig. 1. Interestingly, although Mrk 421 is believed to emit a low VHE flux baseline (Schubnell et al. 1996), the flux was seldom below one Crab Unit (hereafter C.U. corresponding to an integral flux $F_{E>200 \mathrm{GeV}}=1.96 \pm 0.05_{\text {stat }} \times 10^{-10} \mathrm{~cm}^{-2} \mathrm{~s}^{-1}$, Albert et al. 2008a) for the entire period, confirming an intense and persistent active state. The maximum observed flux $\left(F_{E>200 \mathrm{GeV}}=6.99 \pm\right.$ $0.15_{\text {stat }} \times 10^{-10} \mathrm{~cm}^{-2} \mathrm{~s}^{-1}$ ) was on 2008 March 30. Similar fluxes were reached in the flare that occurred in June 2008, which was already studied in detail in Donnarumma et al. (2009). Similar high flux levels during the same period are reported in Acciari et al. (2011), with a record flux of $\sim 12 \times 10^{-10} \mathrm{~cm}^{-2} \mathrm{~s}^{-1}$ above $300 \mathrm{GeV}$ (corresponding to 10 C.U.) observed in May 2008.

The sensitivity of MAGIC-I allowed us to investigate the sub-hour scale evolution of VHE flux for Mrk 421 in high state, searching for the rapid variations already reported in literature 


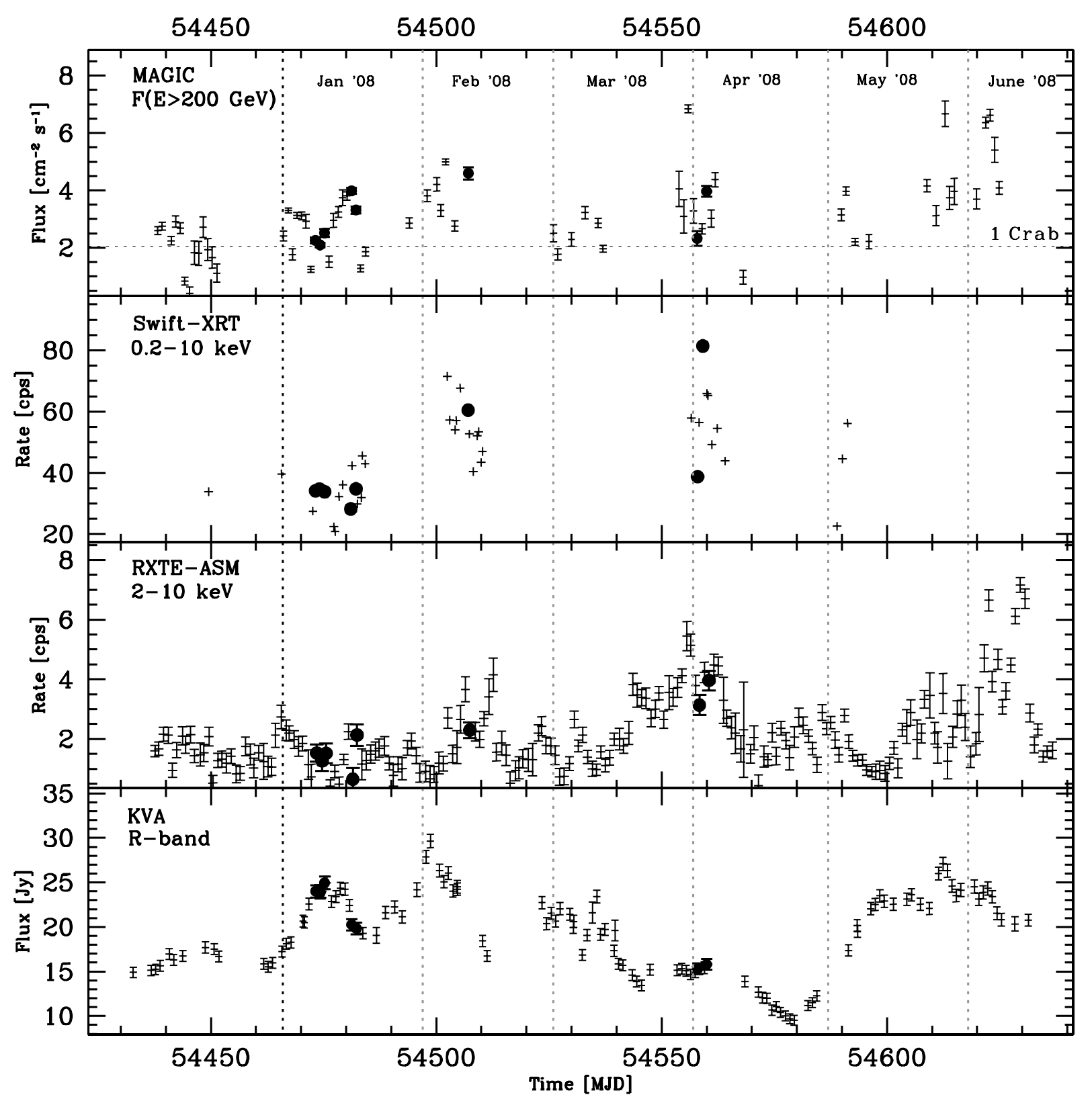

Fig. 1. Multi-wavelength light curves of Mrk 421 along the MAGIC-I observation period; full circles mark the fluxes observed when MAGIC-I and Swift/XRT were pointed at the source simultaneously. Upper panel: MAGIC-I VHE light curve above $200 \mathrm{GeV}$, for the 66 observation nights that passed quality cuts. MAGIC-I detected the source clearly in all nights; the integral flux was below the Crab Unit $\left(\mathrm{C} . \mathrm{U}\right.$., $\approx F_{E>200 \mathrm{GeV}}=2.0 \times$ $10^{-10}$ photons $\mathrm{cm}^{-2} \mathrm{~s}^{-1}$, represented here by the dashed horizontal line) only in a few nights. A maximum flux of $\sim 3.6 \mathrm{C}$.U. was observed on 2008 March the 30th (MJD = 54 555). Middle-upper panel: soft X-ray $(0.2-10 \mathrm{keV})$ count rates measured by Swift/XRT. Middle-lower panel: soft X-ray $(2-10 \mathrm{keV})$ count rates measured by RXTE/ASM. Lower panel: Johnson $R$-band optical light curve from the Tuorla Observatory.

(Gaidos et al. 1996). The most interesting result was obtained on 2008 February 6 , when a long $(\sim 4 \mathrm{~h})$ observation of a high ( 2.5 C.U. above $200 \mathrm{GeV}$ ) state was performed. The VHE light curve in 8-min time bins is shown in Fig. 2, above a softer $(E>200 \mathrm{GeV}$, upper panel) and harder $(E>400 \mathrm{GeV}$, lower panel) energy threshold. An episode of variability with doubling/halving times down to 16 min can be seen with the harder cut. The hypothesis of a steady flux is unfit in both light curves according to results of a $\chi^{2}$ test, giving $\chi^{2} /$ d.o.f. of $55 / 28$ (probability below $0.2 \%$ ) and $63 / 28$ (probability $\sim 10^{-4}$ ), respectively, which confirms variability on the scale of hours or less. Unfortunately, no simultaneous Swift/XRT observation was performed in this night, therefore we could not add this VHE observation to the set of simultaneous MWL SED. No firm conclusion could be drawn on the sub-hour variability for the simultaneous datasets, because some observation windows were very short (e.g. April the 2nd and 3rd) and other were made in lower flux levels (e.g. January 8-10), which led to poorer event statistics.

\subsubsection{Multi-wavelength data}

The Swift-XRT observed count rates in the $0.2-10 \mathrm{keV}$ band are reported in the middle-upper panel of Fig. 1.

The count rates observed by RXTE/ASM in the $2-10 \mathrm{keV}$ band are shown in the middle-lower panel of Fig. 1. 

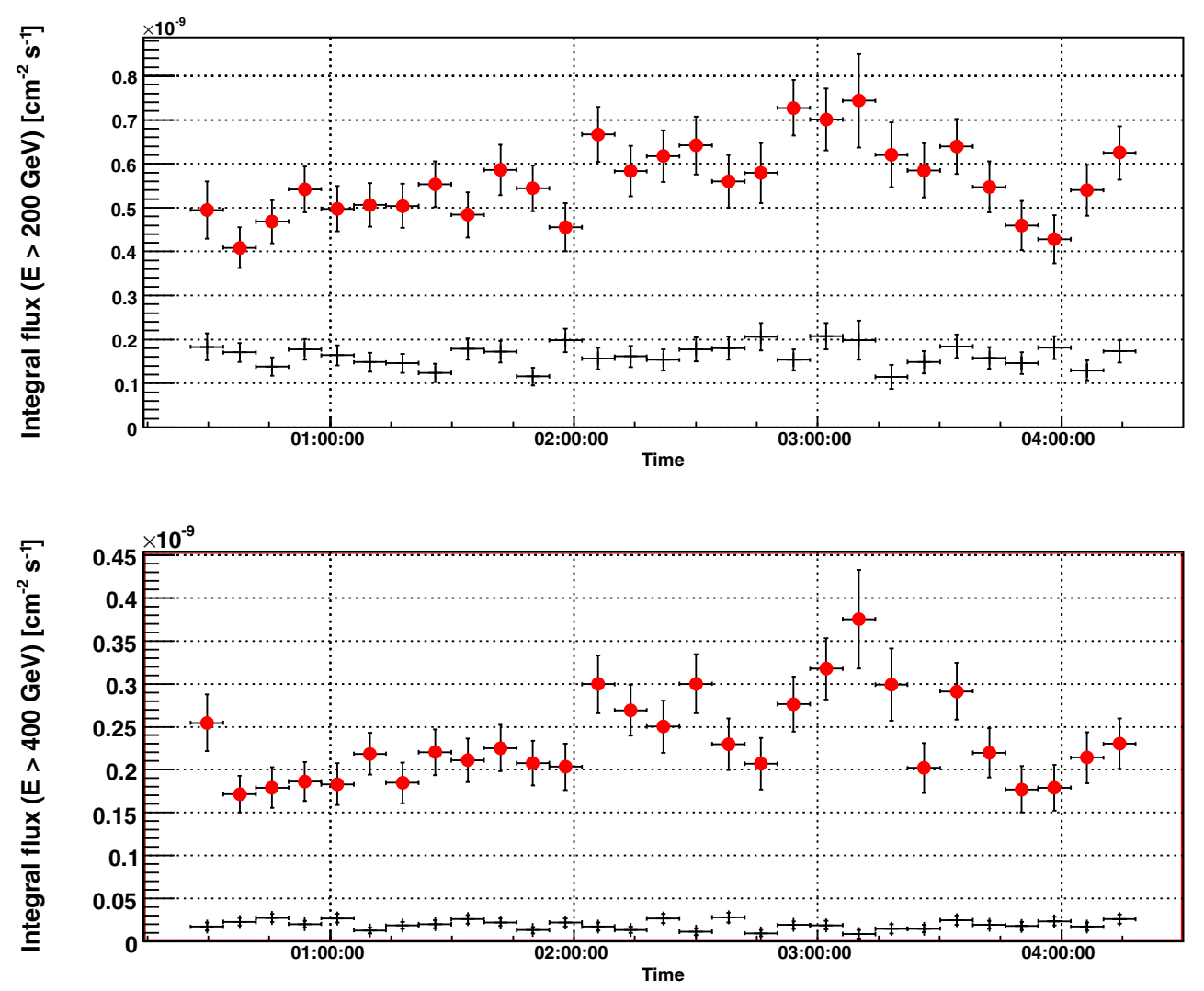

Fig. 2. Mrk 421 VHE light curves in 8-min time bins from the observations taken on 2008 February 6. Integral flux of excess (filled circles) and background (thin crosses) events are plotted. The energy threshold is $200 \mathrm{GeV}$ (upper panel) and $400 \mathrm{GeV}$ (lower panel).

The $R$-band optical light curve from KVA observations is reported in the bottom panel of Fig. 1, while the available measurements related to the simultaneous datasets listed in Table 3 (see Sect. 4) are plotted in Fig. 6 after correction for Galactic extinction, again applied according to the values of Schlegel et al. (1998).

\subsection{VHE spectra derived from MAGIC-I data}

We restricted the study of the spectra to the subset of the eight observations of interest for modeling the MWL SED (see Sect. 4), listed in Table 3. From each observation we derived a VHE spectrum in bins of the estimated energy of the $\gamma$-ray primary events. Then we applied the Tikhonov unfolding algorithm (Albert et al. 2007c) to reconstruct the physical spectrum in terms of the true energy of the primary $\gamma$-rays. A best fit to the data was then performed, assuming a log-parabolic model for the differential spectrum:

$$
\frac{\mathrm{d} N}{\mathrm{~d} E \mathrm{~d} A \mathrm{~d} t}=f_{0} \times\left(\frac{E}{E_{0}}\right)^{\left(a+b \cdot \log \left(\frac{E}{E_{0}}\right)\right)},
$$

where the pivot energy $E_{0}$ is chosen $300 \mathrm{GeV}$ in the present case. In three cases a simple power law was sufficient to fit the data.

For each night, the integral VHE flux above $200 \mathrm{GeV}$, the parameters of the fit to the observed (no EBL correction) emission and the $\tilde{\chi}^{2}$ are reported in Table 1; quoted uncertainties are statistical only.

Adopting the $a$ parameter, giving the slope of the spectrum at the pivot energy, as an estimator of its hardness, it is evident that the well-observed "harder when brighter" trend (see e.g. Acciari et al. 2011; Fossati et al. 2008) is nicely reproduced in these spectra. The spectral points were subsequently corrected for extra-balactic background light (EBL) absorption. The Franceschini et al. (2008) EBL model has been assumed in this work, even if consistent results can be obtained with other models such as the more recent Domínguez et al. (2011) model, given that for this close-by source the model-to-model differences in opacity below $10 \mathrm{TeV}$ are dominated by the statistical uncertainties in hour-scale integrated VHE spectra. The data points are plotted in Fig. 4 along with the SSC models (see Sect. 4.1) that are anticipated here only as a help to guide the eye. For comparison we also plot, without a model, the SED built from the observation achieving the highest VHE flux of the whole campaign (March 30). Unfortunately, we were unable to include this interesting dataset in the SED study, because Swift could only observe with $14 \mathrm{~h}$ of delay with respect to MAGIC-I. Anyway, the VHE spectrum derived from this observation is intriguingly hard, peaking around $500 \mathrm{GeV}$, well within the MAGIC-I band. In Fig. 3 the observed SED (black open triangles) is plotted together with the deabsorbed one (red filled circles), which peaks above $1 \mathrm{TeV}$.

\subsection{Soft $X$-ray spectra derived from Swift/XRT data}

For the eight simultaneous observations with MAGIC-I listed in Table 3 we extracted the Swift/XRT spectra to build the MWL SED (see Sect. 4). Data were rebinned to obtain at least 30 counts per energy bin. Broken power-law models were fitted to the spectra in the range $0.35-10 \mathrm{keV}$. The $\mathrm{X}$-ray reddening due to absorbing systems along the light travel path was corrected assuming the Galactic value for the column density of neutral hydrogen $N_{\mathrm{H}}=1.6 \times 10^{20} \mathrm{~cm}^{-2}$ (Lockman \& Savage 1995). Table 2 reports for each dataset (with uncertainties in 
Table 1. Results from MAGIC-I VHE observations of Mrk 421 during the eight nights with simultaneous Swift/XRT data.

\begin{tabular}{lccccc}
\hline \hline Night & $\begin{array}{c}\text { Integral flux } \\
\mathrm{cm}^{-2} \mathrm{~s}^{-1} \\
{\left[\times 10^{-10}\right]} \\
\text { yyyy-mm-dd } \\
(E>200 \mathrm{GeV})\end{array}$ & $\begin{array}{c}f_{0} \\
\mathrm{~cm}^{-2} \mathrm{~s}^{-1} \mathrm{TeV}^{-1} \\
{\left[\times 10^{-10}\right]} \\
\left(E_{0}=300 \mathrm{GeV}\right)\end{array}$ & & $b$ & $\tilde{\chi}^{2} /$ d.o.f. \\
\hline $2008-01-08$ & $2.13 \pm 0.20$ & $5.9 \pm 0.7$ & $-2.72 \pm 0.12$ & - & \\
$2008-01-09$ & $2.61 \pm 0.11$ & $6.3 \pm 0.3$ & $-2.50 \pm 0.07$ & $-0.44 \pm 0.15$ & $0.40 / 5$ \\
$2008-01-10$ & $2.53 \pm 0.16$ & $7.4 \pm 0.5$ & $-2.42 \pm 0.08$ & $-0.52 \pm 0.20$ & $1.26 / 6$ \\
$2008-01-16$ & $4.42 \pm 0.14$ & $10 \pm 1$ & $-2.25 \pm 0.07$ & $-0.33 \pm 0.10$ & $0.34 / 6$ \\
$2008-01-17$ & $3.80 \pm 0.19$ & $9.8 \pm 1.2$ & $-2.37 \pm 0.10$ & $-0.57 \pm 0.18$ & $0.70 / 6$ \\
$2008-02-11$ & $5.34 \pm 0.32$ & $12 \pm 1$ & $-2.11 \pm 0.14$ & $-0.44 \pm 0.24$ & $1.10 / 6$ \\
$2008-04-02$ & $2.94 \pm 0.32$ & $7.1 \pm 0.5$ & $-2.44 \pm 0.16$ & - & $0.31 / 3$ \\
$2008-04-03$ & $4.53 \pm 0.30$ & $11 \pm 1$ & $-2.35 \pm 0.10$ & - & $0.37 / 6$ \\
\hline
\end{tabular}

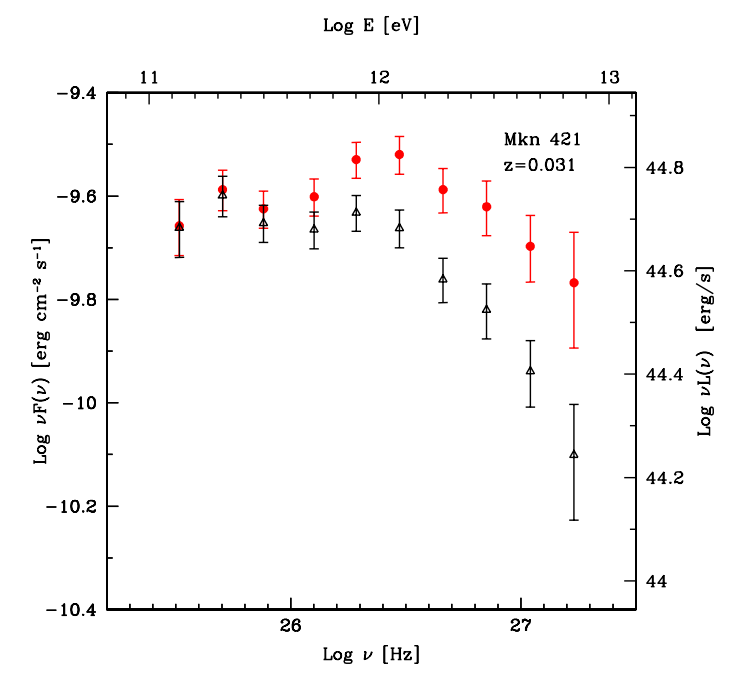

Fig. 3. VHE SED of Mrk 421 derived from the MAGIC-I observations performed on 2008 March 30, when the flux rose to 3.6 Crab Units. Data points before (black open triangles) and after (red filled circles) applying a correction for EBL absorption following Franceschini et al. (2008) are shown. The observed position of the IC peak is evaluated at $\sim 500 \mathrm{GeV}$ from the fit with a curved power law, and above $1 \mathrm{TeV}$ after deabsorption. This VHE spectrum was the hardest among the ones studied here, as illustrated in Fig. 4.

parentheses) the Obs ID, the UTC time at the beginning of observation, the exposure time, the integral flux in the $2-10 \mathrm{keV}$ band, the spectral indexes, break energy and normalization at $1 \mathrm{keV}$ of the broken power law, and the resulting $\tilde{\chi}^{2}$ (reduced $\chi^{2}$ ) with the number of degrees of freedom.

\section{Simultaneous multi-wavelength datasets}

Below we focus on the eight cases for which tightly simultaneous observations in VHE with MAGIC-I telescope and in X-rays with Swift/XRT could be performed. Table 3 summarizes the observation logs of the two instruments for these nights. For each one we report the beginning and the end of the MAGIC-I observation time span, the total effective time, and the ZA range of each dataset. The start time and duration of the corresponding Swift/XRT pointing are also reported, along with the actual overlapped observing time (in ks) in the last column. The MAGIC-I data considered for each night always cover a timespan that is longer than the Swift exposures: this was necessary, because the typical observation time of Swift in this campaign (1 ks) is enough for deriving a fairly detailed X-ray spectrum of
Mrk 421, but the significantly lower count rate available in the $\gamma$-ray domain makes this exposure time too short for deriving a VHE spectrum detailed enough for the modeling. Therefore the whole MAGIC-I exposure was used to derive the VHE spectrum for each night, given that the observing conditions were stable and no evidence for sharp evolution of the source arose from the VHE light curves at minute scales. For each of the eight states we built the MWL SED matching the MAGIC, Swift/XRT and optical-UV (either $R$-band from KVA, or UV from Swift/XRT, or both). As an example, the SED of Mrk 421 as observed on 2008 February 11 is plotted in Fig. 5, compared to historical MWL data taken from Tavecchio \& Ghisellini (2008). It is worth noticing that the VHE SED is high and hard (in agreement with what is expected from other observations of this source during active phases, see e.g. Acciari et al. 2011), while the X-ray SED is high but quite soft compared to past states where the synchrotron peak was observed at higher energies. The wide separation of the two peaks is discussed in detail in Sect. 4.1.

\subsection{SED modeling}

To reduce the degrees of freedom, we used a simple one-zone SSC model (for details see Tavecchio et al. 1998; Maraschi \& Tavecchio 2003), similar to the models commonly adopted to reproduce the SED of Mkn 421 (e.g. Krawczynski et al. 2004; Finke et al. 2008). The emission zone is supposed to be spherical with radius $R$, in motion with bulk Lorentz factor $\Gamma$ at an angle $\theta$ with respect to the line of sight. Special relativistic effects are described by the relativistic Doppler factor, $\delta=[\Gamma(1-\beta \cos \theta)]^{-1}$. The energy distribution of the relativistic emitting electrons is described by a smoothed broken power law function, written for better clarity in terms of the adimensional Lorentz parameter $\gamma=E / m_{\mathrm{e}} c^{2}$; the distribution spans the $\left[\gamma_{\min }, \gamma_{\max }\right]$ energy range, with slopes $n_{1}$ and $n_{2}$ below and above the break energy $\gamma_{\mathrm{b}}$, respectively. This purely phenomenological distribution, with $n_{1}<3$ and $n_{2}>3$, is able to reproduce the observed bumpy SED. To calculate the SSC emission we used the full Klein-Nishina cross section (Jones 1968).

As emphasized in Bednarek \& Protheroe (1997) and Tavecchio et al. (1998), constraints can be put to this simple model by means of simultaneous multi-wavelength observations. Indeed, the total number of free parameters of the model is reduced to nine: the six parameters specifying the electron energy distribution plus the Doppler factor, the size of the emission region and the magnetic field. On the other hand, from X-ray and VHE observations one can ideally derive seven observational quantities: the slopes of the synchrotron bump before and above 
$\log \mathrm{E}[\mathrm{eV}]$

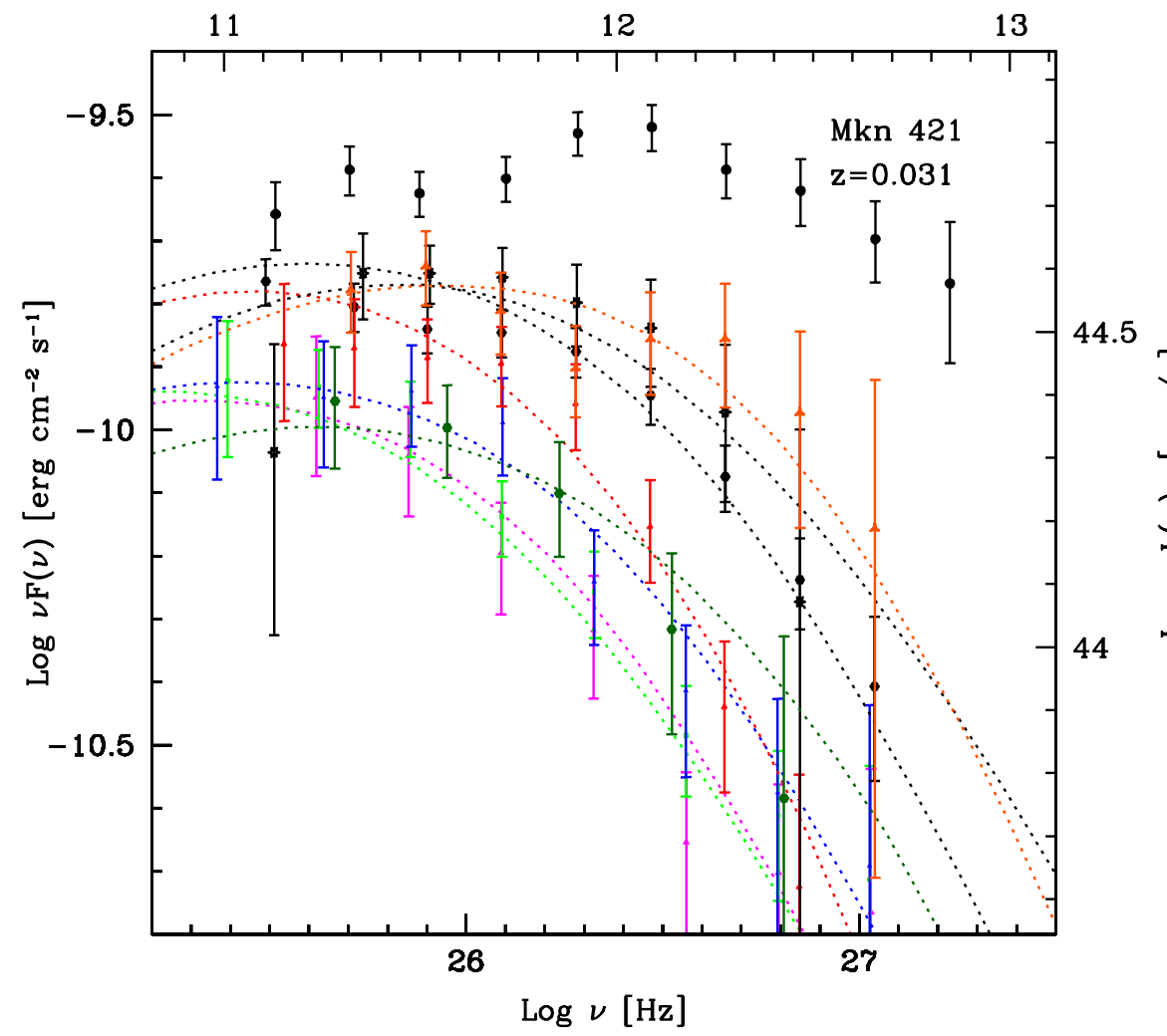

Fig. 4. VHE SED of Mrk 421 derived from the MAGIC-I observations performed in the eight time slots with tightly simultaneous Swift/XRT data (see Sect. 4). The spectra are shown after correction of the EBL absorption, following Franceschini et al. (2008). For comparison, the spectrum derived from the observation that registered the highest flux (3.6 C.U. above $200 \mathrm{GeV}$ ) of the whole campaign, performed on 2008 March 30. For each night we also plot our model (see Sect. 4.1) to guide the eye.

Table 2. Mrk 421 soft X-ray fluxes and spectral parameters from the eight Swift/XRT datasets simultaneous to MAGIC-I observations.

\begin{tabular}{|c|c|c|c|c|c|c|c|c|}
\hline Obs. ID & $\begin{array}{c}\text { Start } \\
\text { Time (UT) } \\
\text { yyyy-mm-dd hh:mm }\end{array}$ & $\begin{array}{l}\text { Obs. } \\
\text { Time } \\
\text { ks }\end{array}$ & $\begin{array}{c}F_{2-10 \mathrm{keV}} \\
\mathrm{erg} / \mathrm{cm}^{2} / \mathrm{s} \\
{\left[\times 10^{-12}\right]}\end{array}$ & $\alpha_{1}$ & $\begin{array}{c}E_{\text {break }} \\
\mathrm{keV}\end{array}$ & $\alpha_{2}$ & $f_{0}$ & $\tilde{\chi}^{2} /$ d.o.f. \\
\hline 00030352041 & 2008-01-08 02:30 & 2.0 & 280 & $2.31(0.03)$ & $1.20(0.09)$ & $2.58(0.03)$ & $0.242(0.003)$ & $1.34 / 163$ \\
\hline 00030352042 & 2008-01-09 04:04 & 2.0 & 283 & $2.28(0.03)$ & $1.05(0.10)$ & $2.60(0.03)$ & $0.257(0.005)$ & $1.30 / 170$ \\
\hline 00030352044 & $2008-01-1002: 27$ & 2.3 & 284 & $2.32(0.02)$ & $1.10(0.10)$ & $2.57(0.02)$ & $0.245(0.003)$ & $1.59 / 178$ \\
\hline 00030352053 & 2008-01-16 03:21 & 1.2 & 345 & $2.19(0.04)$ & $1.24(0.18)$ & $2.45(0.04)$ & $0.242(0.004)$ & $1.16 / 122$ \\
\hline 00030352055 & 2008-01-17 03:29 & 0.8 & 311 & $2.21(0.03)$ & $1.97(-0.18 /+0.4)$ & $2.75(-0.09 /+0.19)$ & $0.243(0.003)$ & $1.33 / 101$ \\
\hline 00030352068 & 2008-02-11 03:40 & 1.9 & 587 & $2.19(0.01)$ & $2.43(0.2)$ & $2.57(0.06)$ & $0.372(0.002)$ & $1.76 / 215$ \\
\hline 00030352083 & 2008-04-02 00:42 & 0.9 & 474 & $2.09(0.02)$ & $2.86(0.33)$ & $2.51(-0.08 /+0.15)$ & $0.260(0.003)$ & $1.47 / 130$ \\
\hline 00030352086 & 2008-04-03 21:59 & 1.2 & 961 & $1.95(0.02)$ & $2.37(-0.16 /+0.28)$ & $2.33(0.06)$ & $0.438(0.003)$ & $1.48 / 210$ \\
\hline
\end{tabular}

the peak $\alpha_{1,2}$ (uniquely connected to $n_{1,2}$ ), the synchrotron and SSC peak frequencies $\left(v_{\mathrm{s}, \mathrm{C}}\right)$ and luminosities $L_{\mathrm{s}, \mathrm{C}}$ and the minimum variability timescale $t_{\mathrm{var}}$, which provides an upper limit to the size of the sources through the relation $R<c t_{\mathrm{var}} \delta$. It must be noted that as long as $\gamma_{\min } \ll \gamma_{\mathrm{b}} \ll \gamma_{\max }$ the values of $\gamma_{\min }$ and $\gamma_{\max }$ are not very constrained by the observation of the peaks. Nevertheless, the availability of the spectral shape across the instrument bandpasses and of data at other wavelengths (optical and UV in this case) provide additional constraints with respect to the simple seven quantities enumerated above. Therefore, once all the observational quantities are known, one can fairly unambiguosly derive the set of parameters. In this respect, the cases studied here are quite favorable, because we have a fairly good determination of the peak frequencies (and fluxes) of both peaks. Indeed, although the synchrotron peak of Mrk 421 is seldom observed within the band encompassed by XRT, the joint optical-UV and X-ray data provide a good constraint to the position of the synchrotron peak in all the cases. The SSC peak is located either within (see e.g. the SED from February 11 in Fig. 5) the MAGIC-I band, or around its lower edge; in the latter case the pronounced curvature of the MAGIC-I spectrum at the lowest energies allows us to constrain the peak at energies not much below $\approx 50 \mathrm{GeV}$.

Unfortunately, for the epochs used to derive the SEDs we do not have information on the variability timescale, $t_{\mathrm{var}}$, one of the key observational parameters needed to completely close the system and uniquely derive the parameters. In the X-ray band Swift/XRT observed in short $(\sim 1 \mathrm{ks})$ snapshots, while no convincing evidence for sub-hour variability arose from the corresponding MAGIC-I observations. Therefore we still have some freedom in choosing the input parameters: one can obtain different sets of parameters that reproduce the spectral data equally well but differ in the predicted observed minimum variability timescale.

We applied the model to all eight sets of data collected when Swift and MAGIC-I could observe the source simultaneously. 
Table 3. Summary of the eight tightly simultaneous observations of Mrk 421 with MAGIC-I and Swift/XRT.

\begin{tabular}{lccccccc}
\hline \hline \multirow{2}{*}{ Night } & \multicolumn{3}{c}{ MAGIC Obs. } & \multicolumn{3}{c}{ Swift/XRT Obs. } & Overlap \\
yyyy-mm-dd & $\begin{array}{c}\text { Start (UT) } \\
\text { (hh.mm) }\end{array}$ & $\begin{array}{c}\text { End (UT) } \\
(\mathrm{hh} . \mathrm{mm})\end{array}$ & $\begin{array}{c}\text { Time } \\
(\mathrm{ks})\end{array}$ & $\begin{array}{c}\text { ZA range } \\
(\mathrm{deg})\end{array}$ & $\begin{array}{c}\text { Start (UT) } \\
(\mathrm{hh} . \mathrm{mm})\end{array}$ & $\begin{array}{c}\text { Time } \\
(\mathrm{ks})\end{array}$ & $\begin{array}{c}\text { Time } \\
(\mathrm{ks})\end{array}$ \\
\hline $2008-01-08$ & 01.58 & 02.44 & 2.7 & $31-41$ & 02.30 & 2.0 & 0.8 \\
$2008-01-09$ & 03.56 & 06.19 & 8.2 & $6-20$ & 04.04 & 2.0 & 2.0 \\
$2008-01-10$ & 02.23 & 06.05 & 3.5 & $10-34$ & 02.27 & 2.3 & 1.1 \\
$2008-01-16$ & 03.17 & 05.11 & 6.5 & $6-21$ & 03.21 & 1.2 & 1.2 \\
$2008-01-17$ & 03.26 & 04.25 & 3.4 & $6-18$ & 03.29 & 0.8 & 0.8 \\
$2008-02-11$ & $03: 33$ & $03: 58$ & 1.4 & $10-18$ & 03.40 & 1.9 & 1.1 \\
$2008-04-02$ & 00.45 & 01.00 & 0.9 & $17-21$ & 00.42 & 0.9 & 0.6 \\
$2008-04-03$ & 21.55 & 22.20 & 1.3 & $15-23$ & 21.59 & 1.2 & 1.1 \\
\hline
\end{tabular}

\section{$\log \mathrm{E}[\mathrm{eV}]$}

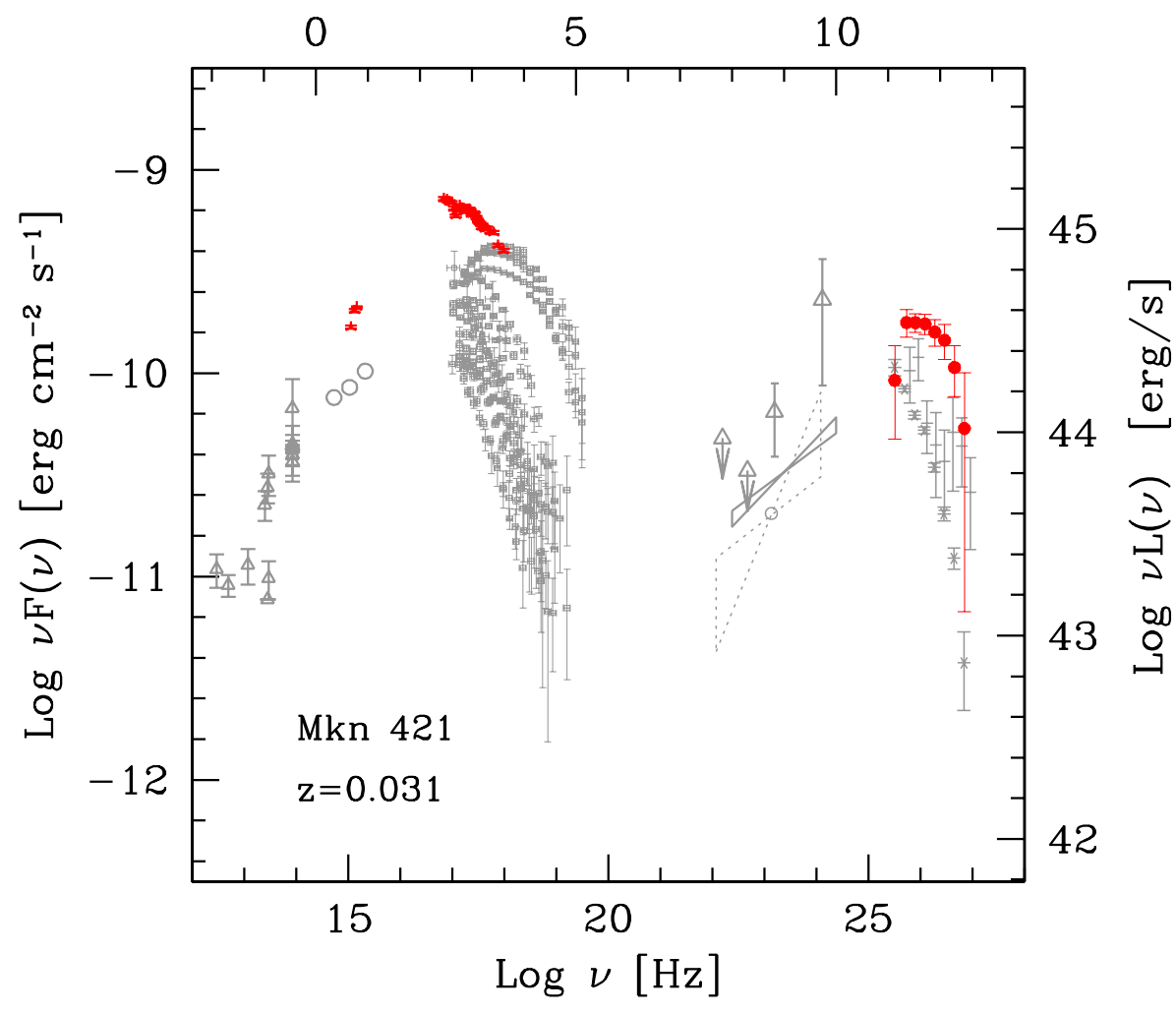

Fig. 5. Example MWL SED of Mrk 421 as observed in tight simultaneity by Swift/UVOT, Swift/XRT and MAGIC-I on 2008 February 11 (filled red circles). Historical data (observed in different campaigns, and under less demanding time constraints) taken from Tavecchio et al. (2010) are plotted for comparison, with gray open symbols.

UVOT and KVA data were also included in the SED when available. The sets of parameters obtained from the modeling are reported in Table 4 and the SED data and the corresponding model are plotted in Fig. 6. The table reports for each night the minimum $\left(\gamma_{\min }\right)$, break $\left(\gamma_{\mathrm{b}}\right)$ and maximum $\left(\gamma_{\max }\right)$ Lorentz factors of the electron distribution, the low $\left(n_{1}\right)$ and high $\left(n_{2}\right)$ energy slopes of the electron distribution, the magnetic field $(B)$ and the electron normalization $(K)$ within the emitting region, the radius of the emitting region $(R)$ and its Doppler factor $(\delta)$. From these input parameters we derived the light crossing time $\left(t_{\mathrm{var}}\right)$; the contributions to the total jet luminosity from cold protons $\left(L_{\mathrm{kin}}^{\mathrm{p}}\right)$ and relativistic electrons $\left(L_{\mathrm{kin}}^{\mathrm{e}}\right)$ in the jet, and from magnetic field $\left(L_{\mathrm{B}}\right)$; the electron $\left(u_{\mathrm{e}}\right)$ and magnetic $\left(u_{\mathrm{B}}\right)$ energy densities. We note that in reproducing the SED we did not consider the radio data, since the modeled region is opaque at these frequencies: in this framework the radio emission originates in regions of the jet farther away from the black hole, beyond the core visible at VLBI scale, which is thought to mark the radio "photosphere".
Accordingly, the inferred source radius is well within the upper limit of $0.1 \mathrm{pc}\left(3 \times 10^{17} \mathrm{~cm}\right)$ imposed by Charlot et al. (2006) for the projected size of the SSC zone, based on VLBI observations of the radio core.

Inspection of Table 4 shows that the derived Doppler factors are quite high, exceeding $\delta=40$ in all the cases and reaching values as high as $80-85$ in the most extreme cases. The main reason for these high values of $\delta$ is the large separation between the two peaks, the synchrotron one located below $10^{17} \mathrm{~Hz}$, the SSC one around $10^{25} \mathrm{~Hz}$ or above. As detailed in, e.g., Tavecchio \& Ghisellini (2008), a large distance between the two peaks implies a fairly high value of the Lorentz factor at the peak, $\gamma_{\mathrm{b}}$, since $\gamma_{\mathrm{b}}=\left(v_{\mathrm{C}} / v_{\mathrm{s}}\right)^{1 / 2}$, and this directly implies a low $B$ and a large $\delta$ to satisfy the other constraints.

However, we recall that because the variability timescale is not known, we are left with some freedom in selecting the input parameters. In the models reported in Fig. 6 we assumed variability timescales in the range $0.5-2 \mathrm{~h}$, as typically derived 
Table 4. Input model parameters and derived physical quantities for each of the eight simultaneous SED.

\begin{tabular}{lccccccccccccccc}
\hline \hline $\begin{array}{l}\text { Night } \\
\text { yyyy-mm-dd }\end{array}$ & $\begin{array}{c}\gamma_{\min } \\
{\left[\times 10^{3}\right]}\end{array}$ & $\begin{array}{c}\gamma_{\mathrm{b}} \\
{\left[\times 10^{4}\right]}\end{array}$ & $\begin{array}{c}\gamma_{\max } \\
{\left[\times 10^{6}\right]}\end{array}$ & $n_{1}$ & $n_{2}$ & $\begin{array}{c}B \\
{[G]}\end{array}$ & $\begin{array}{c}K \\
{\left[\mathrm{~cm}^{-3}\right]}\end{array}$ & $\begin{array}{c}R \\
{\left[\times 10^{15} \mathrm{~cm}\right]}\end{array}$ & $\delta$ & $\begin{array}{c}t_{\mathrm{var}} \\
{[\mathrm{h}]}\end{array}$ & $\begin{array}{c}L_{\text {kin }}^{p} \\
{\left[\times 10^{42} \mathrm{erg} / \mathrm{s}\right]}\end{array}$ & $\begin{array}{c}L_{\mathrm{B}}^{\mathrm{e}} \\
{\left[\times 10^{-5} \mathrm{erg} / \mathrm{cm}^{3}\right]}\end{array}$ \\
\hline $2008-01-08$ & 7.0 & 6.0 & 3.0 & 2.0 & 4.0 & 0.050 & 1700 & 9.0 & 45 & 1.8 & 5.41 & 91 & 1.61 & 420 & 9.9 \\
$2008-01-09$ & 10 & 2.9 & 3.0 & 2.0 & 4.0 & 0.043 & 3700 & 5.0 & 85 & 0.5 & 7.37 & 136 & 1.25 & 600 & 7.4 \\
$2008-01-10$ & 6.0 & 5.7 & 3.0 & 2.0 & 4.0 & 0.037 & 3300 & 5.0 & 70 & 0.7 & 8.83 & 131 & 0.63 & 850 & 5.4 \\
$2008-01-16$ & 8.3 & 6.7 & 3.0 & 2.0 & 4.0 & 0.025 & 4000 & 5.0 & 80 & 0.6 & 9.97 & 197 & 0.38 & 980 & 2.5 \\
$2008-01-17$ & 10 & 6.0 & 0.7 & 2.0 & 4.2 & 0.037 & 2600 & 7.2 & 60 & 1.1 & 6.18 & 138 & 0.96 & 590 & 5.4 \\
$2008-02-11$ & 11 & 6.9 & 3.0 & 2.0 & 3.7 & 0.020 & 2400 & 6.6 & 85 & 0.7 & 6.86 & 187 & 0.47 & 470 & 1.6 \\
$2008-04-02$ & 8.0 & 3.2 & 1.0 & 2.0 & 3.5 & 0.050 & 5900 & 3.9 & 70 & 0.5 & 5.24 & 80 & 0.46 & 1200 & 9.2 \\
$2008-04-03$ & 17 & 20 & 3.0 & 2.0 & 4.0 & 0.040 & 2000 & 8.5 & 40 & 2.0 & 5.47 & 120 & 0.62 & 520 & 3.6 \\
\hline
\end{tabular}

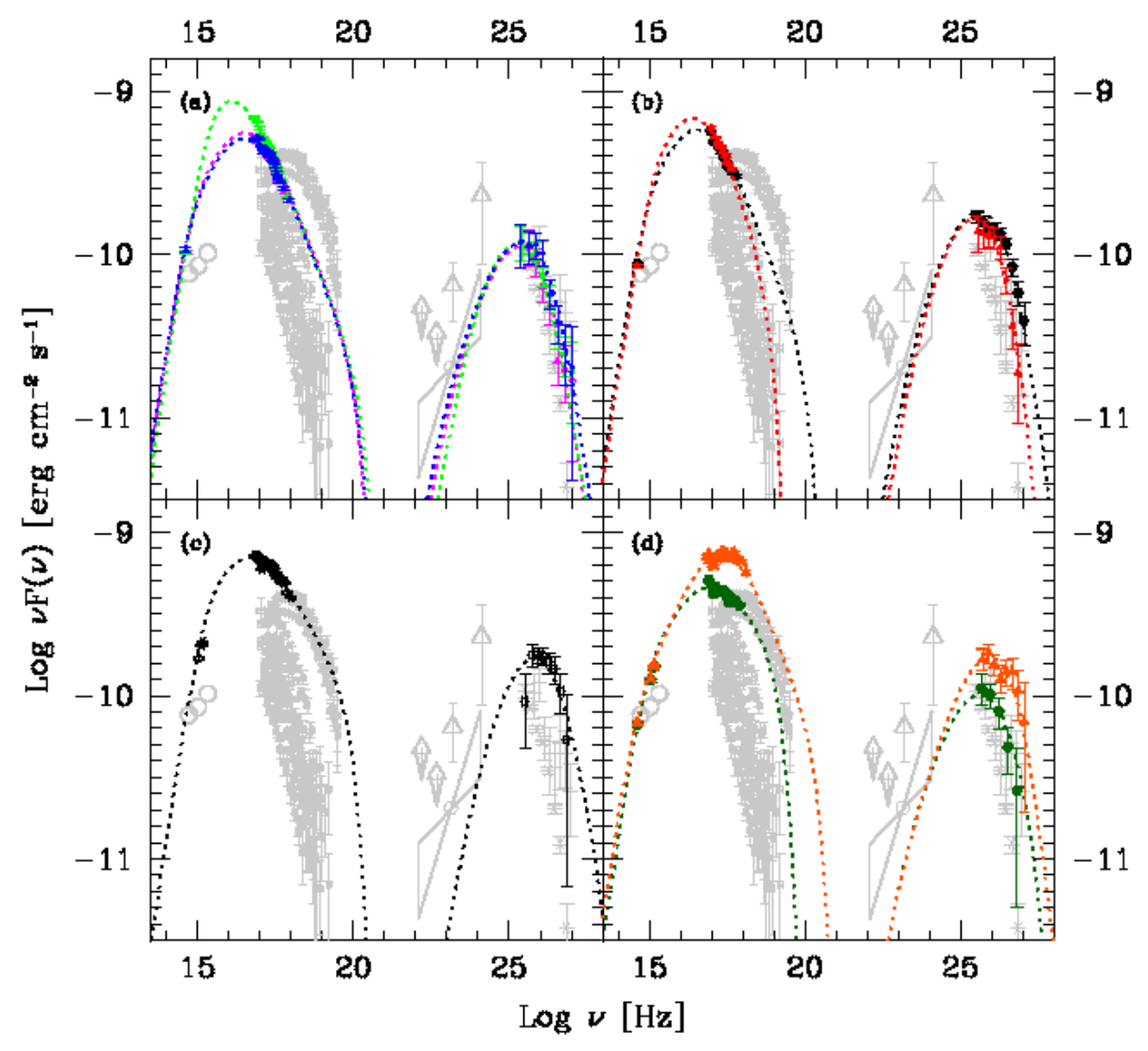

Fig. 6. SED of Mrk 421 with the SSC model overplotted for each of the eight simultaneous sets of MAGIC-I, Swift/XRT and optical-UV data obtained in the 2008 campaign. Observation dates are January $8-10$ a), January 16 and 17 b), February 11 c) and April 2 and 3 d). for these sources (see discussion below). In general, the required Doppler factor roughly scales with the observed variability timescale as $\delta \propto t_{\text {var }}^{-0.5}$ (e.g. Tavecchio \& Ghisellini 2008). Therefore, relaxing the condition on $t_{\mathrm{var}}$ and allowing longer minimum variability timescales, one obtains lower $\delta$. As an example we used the case for which we derive the largest $\delta$, that of February 11, requiring $\delta=85$. As noted above, here the determination of the peak frequencies is very robust, because the SSC peak falls well within the band covered by MAGIC-I. Therefore this is also the best "benchmark" available to test the robustness of the derived parameters. For this purpose we modeled this SED assuming two sets of parameters, basically differing for the value of the Doppler factor, the radius of the emitting region and the magnetic field intensity. For $\delta=85$ we have $t_{\mathrm{var}}=0.7 \mathrm{~h}\left(2.5 \times 10^{3} \mathrm{~s}\right)$, while more than halving the Doppler factor $\delta=40$ implies a fairly long variability timescale, $t_{\mathrm{var}}=5 \mathrm{~h}\left(1.8 \times 10^{4} \mathrm{~s}\right)$, already longer than the characteristic variability timescale of Mrk 421 in the X-ray band. We can conclude that for the case of February 11, although the parameters cannot be uniquely fixed, the required Doppler factor is high, at least higher than $\delta \approx 40$. All other cases are similar. The derived light crossing times are within the $0.5-2 \mathrm{~h}$ interval. This hypothesis matches well the observed typical raising/decaying timescales of flares of Mrk 421 and similar HBLs (PKS 2155-304, Mrk 501), which are characterized by doubling/halving times of $\approx 10^{4} \mathrm{~s}$ (e.g. Fossati et al. 2008; Ravasio et al. 2004; Zhang 2002; Tanihata et al. 2000), with evidence for the occurrence of even faster events (e.g. Gaidos et al. 1996; Cui 2004).

However, if one relaxes this assumption on the variability timescales, the required Doppler factors remain high. For the data from February 11, which allow a quite robust constraint due to direct observation of both the synchrotron and SSC peak, this implies $\delta>45$. In Table 4 we also report the derived powers carried by the different jet components, namely cold protons $\left(L_{\mathrm{kin}}^{\mathrm{p}}\right)$, relativistic electrons $\left(L_{\mathrm{kin}}^{\mathrm{e}}\right)$ and magnetic field $\left(L_{\mathrm{B}}\right)$, assuming a composition of one cold proton per relativistic electron. Finally, we computed the electron $\left(u_{\mathrm{e}}\right)$ and magnetic $\left(u_{\mathrm{B}}\right)$ energy densities. The jet appears to be strongly matter-dominated, as predicted in the standard picture of HBL sources, and in good 
agreement with the result of the modeling performed in Acciari et al. (2011).

\section{Discussion}

During the 2008 campaign on Mrk 421 with MAGIC-I a very interesting dataset was gathered in VHE $\gamma$-rays, complemented by crucial data in optical-UV and soft X-rays. For the first time it was possible to collect data in these bands in close simultaneity during high states of the source, so that the derived spectra sampled the SED close to the synchrotron and IC peaks. In this situation the parameters describing the source in the framework of the standard one-zone leptonic model can be determined with remarkable robustness. One of the most relevant results of our analysis is that to reproduce the observed SED with this model, very high Doppler factors are required. There is some freedom in choosing the parameters, mainly because of the not known variability timescale at those epochs. In the models summarized in Table 4 and reported in Fig. 6 we assumed variability timescales in the range of $0.5-2 \mathrm{~h}$.

Indeed, these high values of inferred $\delta$ are not rare: very high Doppler factors, sometimes higher than $\delta \sim 50$, for Mrk 421 and other well-observed HBLs were obtained in the past, leading to the so called " $\delta$-crisis" (e.g. Krawczynski et al. 2002; Acciari et al. 2011; Konopelko et al. 2003; Georganopoulos \& Kazanas 2003; Henri \& Saugé 2006; Giebels et al. 2007; Finke et al. 2008). Analogously, the recent exceptional VHE flare of PKS 2155-304 (Aharonian et al. 2007) seems to require extreme Doppler factors in the framework of one-zone models (Begelman et al. 2008; Ghisellini \& Tavecchio 2008; Finke et al. 2008; Kusunose \& Takahara 2006). These high values of $\delta$ (implying a similarly high value of the bulk Lorentz factors) contrast with the very low jet velocities inferred at VLBI scales in a large part of TeV BL Lacs (e.g. Giroletti et al. 2004; Piner \& Edwards 2004), including Mrk 421, and with the value of $\Gamma$ required from the unification of BL Lacs and FRI radiogalaxies (e.g. Georganopoulos \& Kazanas 2003; Henri \& Saugé 2006).

In addition to the extreme Doppler factor, we can identify two other problems afflicting the standard model, namely the huge difference between the magnetic and particle energy densities and the extremely long cooling times of the emitting relativistic electrons.

Table 4 shows that in all cases the inferred electron energy density substantially exceeds the corresponding magnetic energy density, by up to two orders of magnitude and even more. This result is also generally found from SSC fits of HBL SEDs (see e.g. Acciari et al. 2011, for a recent example), while for other classes of blazars, in particular for FSRQs, equipartition is usually found (e.g. Ghisellini et al. 2010). This evidence disagrees with the general expectations of the diffusive shock acceleration models, in which a substantial equipartition between particles and magnetic field is expected.

Concerning the second problem, namely the long cooling timescales, we point out that, following for instance the formulae in Bednarek \& Protheroe (1997) or Tavecchio et al. (1998), fairly long cooling times $t_{\text {cool }}$, on the order $10^{6} \mathrm{~s}$ in the observer's frame, can be computed from the model parameters in Table 4. Therefore, the declining part of flares cannot be attributed to the cooling of the emitting electrons. A possibility is that adiabatic expansion, which allows quenching of the flux within scales of $R / c$, has to be invoked as one of the viable processes that may explain the observed descent of $\mathrm{TeV}$ and $\mathrm{X}$-ray fluxes on hour scales. But it must be noted that this explanation has the significant drawback of implying a very energetically inefficient jet. A problem related to the long cooling timescale is that one cannot interpret the observed break in the underlying electron energy distribution as the separation between fast and slow cooling particles (e.g. Tavecchio et al. 1998), so it has to be, for instance, assumed to be intrinsic to the injected population. These problems could therefore hint at the unsuitability of the one-zone model for this source.

A solution of these problems faced by the standard one-zone SSC scenario, extensively discussed in literature, is based on the possible existence of multiple active emitting regions (e.g. Błażejowski et al. 2005; Georganopoulos \& Kazanas 2003), based on the possibility that the flow is characterized by portions moving at different speeds. If these regions emit, in each of them the electrons can scatter not only the locally-produced synchrotron photons, but also the soft photons produced in the other region. Moreover, the energy density of these "external" photons is amplified in the rest frame of the emitting region through the relative speed between the two portions of the flow. The result is that the inverse Compton emission of each region is amplified with respect to the SSC emission. As a consequence, the Doppler factor required to reproduce the SED is lower than that of the one-zone model. In particular, in the "spine-layer" model of Ghisellini et al. (2005), it is assumed that the jet has an inner faster core (the spine) that is surrounded by a slower layer. At a narrow angle of view, which is characteristic for blazars, the emission is dominated by the faster spine whose IC emission is a mixture of SSC and "external" Compton components.

This model would also more easily accomodate the short variability time scales observed in Mrk 421, which are hardly explained within the one-zone model due to the long electron cooling times, as discussed in Sect. 4.1. Indeed, in general a 10 times more intense $B$ can be adopted when modeling a given SED (see e.g. Ghisellini et al. 2005); because the synchrotron cooling time scales as $t_{\text {sync }} \propto B^{-2}$, this could lead to cooling times on the order of the required variability time scale.

An alternative scenario that is possibly able to solve these problems is the "minijets" model advocated by Giannios et al. $(2009,2010)$. In this framework, the emission is thought to occur in very fast small portions of plasma resulting from the rapid reconnection of magnetic field lines inside the main jet flow. If the magnetization (ratio of magnetic over kinetic jet power) is high enough, the Lorentz factor of these blobs in the rest frame of the jet can be as high as $\Gamma=50$. Moreover, since the emitting plasma is the residual of the annihilation of magnetic field, one naturally expects a low magnetic energy density and thus a high particle over magnetic energy density ratio.

A modeling of the SED with the more complex (and less constrained) models mentioned above is beyond the scope of this paper and left to future work.

Acknowledgements. We would like to thank the Instituto de Astrofísica de Canarias for the excellent working conditions at the Observatorio del Roque de los Muchachos in La Palma. The support of the German BMBF and MPG, the Italian INFN, the Swiss National Fund SNF, and the Spanish MICINN is gratefully acknowledged. This work was also supported by the Marie Curie program, by the CPAN CSD2007-00042 and MultiDark CSD2009-00064 projects of the Spanish Consolider-Ingenio 2010 programme, by grant DO02-353 of the Bulgarian NSF, by grant 127740 of the Academy of Finland, by the YIP of the Helmholtz Gemeinschaft, by the DFG Cluster of Excellence "Origin and Structure of the Universe", and by the Polish MNiSzW grant 745/N-HESS-MAGIC/2010/0. We acknowledge insightful and constructive criticism from the anonymous referee. 


\section{References}

Acciari, V. A., Aliu, E., Arlen, T., et al. 2011, ApJ, 738, 25

Aharonian, F., Akhperjanian, A. G., Bazer-Bachi, A. R., et al. 2007, ApJ, 664, L71

Albert, J., Aliu, E., Anderhub, H., et al. (the MAGIC Collaboration) 2007a, ApJ, 663,125

Albert, J., Aliu, E., Anderhub, H., et al. 2007b, ApJ, 669, 862

Albert, J., Aliu, E., Anderhub, H., et al. 2007c, Nucl. Inst. Meth. Phys. Res. A, 583,494

Albert, J., Aliu, E., Anderhub, H., et al. (the MAGIC Collaboration) 2008a, ApJ, 674,1037

Albert, J., Aliu, E., Anderhub, H., et al. (the MAGIC Collaboration), 2008b, Nucl. Instr. Meth. A, 588, 424

Albert, J., Aliu, E., Anderhub, H., et al. (the MAGIC Collaboration) 2008c, Nucl. Instr. Meth. A, 594, 407

Aliu, E., Anderhub, H., Antonelli, L. A., et al. (the MAGIC Collaboration), 2009, Astropart. Phys., 30, 293

Baixeras, C., Bastieri, D., Bigongiari, C., et al. 2004, Nucl. Inst. Meth. Phys. Res. A, 518, 188

Barthelmy, S. D., Barbier, L. M., Cummings, J. R., et al. 2005, Space Sci. Rev., 120,143

Bednarek, W., \& Protheroe, R. J. 1997, MNRAS, 292, 646

Begelman, M. C., Fabian, A. C., \& Rees, M. J. 2008, MNRAS, 384, L19

Błażejowski, M., Blaylock, G., Bond, I. H., et al. 2005, ApJ, 630, 130

Bloom, S. D., \& Marscher, A. P. 1996, ApJ, 461, 657

Bradt, H. V., Rothschild, R. E., \& Swank, J. H. 1993, A\&AS, 97, 355

Breiman, L. 2001, Machine Learning, 45, 4

Brinkmann, W., Papadakis, I. E., Raeth, C., Mimica, P., \& Haberl, F. 2005, A\&A, 443, 397

Burrows, D. N., Hill, J. E., Nousek, J. A., et al. 2005, Space Sci. Rev., 120, 165

Charlot, P., Gabuzda, D. C., Sol, H., Degrange, B., \& Piron, F. 2006, A\&A, 457, 455

Cortina, J., Armada, A., Biland, A., et al. 2005, Int. Cosmic Ray Conf., 5, 359

Cui, W. 2004, ApJ, 605, 662

de Vaucouleurs, G., de Vaucouleurs, A., Corwin, H. G., Jr., et al. 1991,

Third Reference Catalogue of Bright Galaxies, Vol. 1-3, XII, 2069 (Berlin Heidelberg New York: Springer-Verlag), 71

Dermer, C. D., \& Schlickeiser, R. 1993, ApJ, 416, 458

Domínguez, A., Primack, J. R., Rosario, D. J., et al. 2011, MNRAS, 410, 2556

Donnarumma, I., Vittorini, V., Vercellone, S., et al. 2009, ApJ, 691, L13

Finke, J. D., Dermer, C. D., \& Böttcher, M. 2008, ApJ, 686, 181

Fomin, V. P., Stepanian, A. A., Lamb, R. C., et al. 1994, Astropart. Phys., 2, 137

Fossati, G., Buckley, J. H., Bond, I. H., et al. 2008, ApJ, 677, 906

Franceschini, A., Rodighiero, G., \& Vaccari, M. 2008, A\&A, 487, 837

Gaidos, J. A., Akerlof, C. W., Biller, S., et al. 1996, Nature, 383, 319

Gehrels, N., Chincarini, G., Giommi, P., et al. 2004, ApJ, 611, 1005

Georganopoulos, M., \& Kazanas, D. 2003, ApJ, 594, L27

Ghisellini, G., \& Tavecchio, F. 2008, MNRAS, 386, L28

Ghisellini, G., Tavecchio, F., \& Chiaberge, M. 2005, A\&A, 432, 401

Ghisellini, G., Tavecchio, F., Foschini, L., et al. 2010, MNRAS, 402, 497

Giannios, D., Uzdensky, D. A., \& Begelman, M. C. 2009, MNRAS, 395, L29

Giannios, D., Uzdensky, D. A., \& Begelman, M. C. 2010, MNRAS, 402, 1649

Giebels, B., Dubus, G., \& Khélifi, B. 2007, A\&A, 462, 29

Giroletti, M., Giovannini, G., Taylor, G. B., \& Falomo, R. 2004, ApJ, 613, 752

Goebel, F., Bartko, H., Carmona, E., et al. 2008, International Cosmic Ray Conference, 3, 1481

Henri, G., \& Saugé, L. 2006, ApJ, 640, 185

Hillas, A. M. 1985, International Cosmic Ray Conference, 3, 445

Hollander, M., \& Wolfe, D. A. 1973, Nonparametric statistical methods (Wiley)

Hsu, C. C., Satalecka, K., Thom, M., et al. 2009 [arXiv: 0907 . 0893]

Jones, F. C. 1968, Phys. Rev., 167, 1159

Konopelko, A., Mastichiadis, A., Kirk, J., de Jager, O. C., \& Stecker, F. W. 2003, ApJ, 597, 851

Krawczynski, H., Coppi, P. S., \& Aharonian, F. 2002, MNRAS, 336, 721

Krawczynski, H., Hughes, S. B., Horan, D., et al. 2004, ApJ, 601, 151

Krennrich, F., Bond, I. H., Bradbury, S. M., et al. 2002, ApJ, 575, L9

Kusunose, M., \& Takahara, F. 2006, ApJ, 651, 113

Li, T.-P., \& Ma, Y.-Q. 1983, ApJ, 272, 317

Lockman, F. J., \& Savage, B. D. 1995, ApJS, 97, 1

Mankuzhiyil, N., Ansoldi, S., Persic, M., \& Tavecchio, F. 2011, ApJ, 733, 14

Mannheim, K. 1993, A\&A, 269, 67

Maraschi, L., \& Tavecchio, F. 2003, ApJ, 593, 667

Maraschi, L., Ghisellini, G., \& Celotti, A. 1992, ApJ, 397, L5

Maraschi, L., Fossati, G., Tavecchio, F., et al. 1999, ApJ, 526, L81

Mücke, A., Protheroe, R. J., Engel, R., Rachen, J. P., \& Stanev, T. 2003, APh, 18,593
Nilsson, K., Pasanen, M., Takalo, L. O., et al. 2007, A\&A, 475, 199

Padovani, P. 2007, Ap\&SS, 309, 63

Padovani, P., \& Giommi, P. 1995, ApJ, 444, 567

Pei, Y. C. 1992, ApJ, 395, 130

Pichel, A. 2009, for the VERITAS Collaboration [arXiv : 0908.0010]

Piner, B. G., \& Edwards, P. G. 2004, ApJ, 600, 115

Poole, T. S., Breeveld, A. A., Page, M. J., et al. 2008, MNRAS, 383, 627

Punch, M., Akerlof, C. W., Cawley, M. F., et al. 1992, Nature, 358, 477

Ravasio, M., Tagliaferri, G., Ghisellini, G., \& Tavecchio, F. 2004, A\&A, 424, 841

Roming, P. W. A., Kennedy, T. E., Mason, K. O., et al. 2005, Space Sci. Rev., 120,95

Schlegel, D. J., Finkbeiner, D. P., \& Davis, M. 1998, ApJ, 500, 525

Schubnell, M. S., Akerlof, C. W., Biller, S., et al. 1996, ApJ, 460, 644

Sikora, M., Stawarz, Ł., Moderski, R., Nalewajko, K., \& Madejski, G. M. 2009, ApJ, 704, 38

Takahashi, T., Kataoka, J., Madejski, G., et al. 2000, ApJ, 542, L105

Tanihata, C., Takahashi, T., Kataoka, J., et al. 2000, ApJ, 543, 124

Tavecchio, F., \& Ghisellini, G. 2008, MNRAS, 385, L98

Tavecchio, F., Maraschi, L., \& Ghisellini, G. 1998, ApJ, 509, 608

Tavecchio, F., Ghisellini, G., Ghirlanda, G., Foschini, L., \& Maraschi, L. 2010, MNRAS, 401, 1570

Tescaro, D., Bartko, H., Galante, N., et al. 2008, International Cosmic Ray Conference, 3, 1393

Tramacere, A., Massaro, F., \& Cavaliere, A. 2007, A\&A, 466, 521

Tramacere, A., Giommi, P., Perri, M., Verrecchia, F., \& Tosti, G. 2009, A\&A, 501,879

Urry, C. M., \& Padovani, P. 1995, PASP, 107, 803

Ushio, M., Tanaka, T., Madejski, G., et al. 2009, ApJ, 699, 1964

Wagner, R., Backes, M., Satalecka, K., et al. 2011 [arXiv: 1109.6774]

Zhang, Y. H. 2002, MNRAS, 337, 609

1 IFAE, Edifici Cn., Campus UAB, 08193 Bellaterra, Spain

2 Universidad Complutense, 28040 Madrid, Spain

3 INAF National Institute for Astrophysics, 00136 Rome, Italy e-mail: [giacomo.bonnoli; fabrizio.tavecchio] @brera.inaf.it

4 Università di Siena, and INFN Pisa, 53100 Siena, Italy e-mail: antonio.stamerra@pi.infn.it

5 Technische Universität Dortmund, 44221 Dortmund, Germany

6 Università di Padova and INFN, 35131 Padova, Italy

7 Inst. de Astrofísica de Canarias, 38200 La Laguna, Tenerife, Spain

8 Depto. de Astrofísica, Universidad de La Laguna, 38206 La Laguna, Spain

9 University of Łódź, 90236 Lodz, Poland

10 Tuorla Observatory, University of Turku, 21500 Piikkiö, Finland

11 Deutsches Elektronen-Synchrotron (DESY), 15738 Zeuthen, Germany

12 ETH Zurich, 8093, Switzerland

13 Max-Planck-Institut für Physik, 80805 München, Germany

14 Universität Würzburg, 97074 Würzburg, Germany

15 Universitat de Barcelona (ICC/IEEC), 08028 Barcelona, Spain

16 Università di Udine, and INFN Trieste, 33100 Udine, Italy

17 Institut de Ciències de l'Espai (IEEC-CSIC), 08193 Bellaterra, Spain

18 Inst. de Astrofísica de Andalucía (CSIC), 18080 Granada, Spain

19 Croatian MAGIC Consortium, Rudjer Boskovic Institute, University of Rijeka and University of Split, 10000 Zagreb, Croatia

20 Universitat Autònoma de Barcelona, 08193 Bellaterra, Spain

21 Inst. for Nucl. Research and Nucl. Energy, 1784 Sofia, Bulgaria

22 INAF/Osservatorio Astronomico and INFN, 34143 Trieste, Italy

23 Università dell'Insubria, Como, 22100 Como, Italy

24 Università di Pisa, and INFN Pisa, 56126 Pisa, Italy

25 ICREA, 08010 Barcelona, Spain

26 Now at École polytechnique fédérale de Lausanne (EPFL), Lausanne, Switzerland

27 Supported by INFN Padova

28 Now at: Centro de Investigaciones Energéticas, Medioambientales y Tecnológicas (CIEMAT), Madrid, Spain

29 Now at: Finnish Centre for Astronomy with ESO (FINCA), University of Turku, Finland 